\title{
On the flexural vibration of cylinders under axial loads: Numerical and experimental study
}

\author{
A. Bayón ${ }^{\text {a }}$, F. Gascón ${ }^{\text {b }}$, R. Medina ${ }^{\text {a, F.J. Nieves }}{ }^{\text {b }}$, F.J. Salazar ${ }^{\text {a,* }}$ \\ a Departamento de Fisica Aplicada a los Recursos Naturales, ETSI Minas, Universidad Politécnica de Madrid, Ríos Rosas 21, 28003 Madrid, Spain \\ ${ }^{\mathrm{b}}$ Departamento de Fisica Aplicada II, ETS de Arquitectura, Universidad de Sevilla, Reina Mercedes 2, 41012 Sevilla, Spain
}

\section{Introduction}

\begin{abstract}
A B S T R A C T
The flexural vibration of a homogeneous isotropic linearly elastic cylinder of any aspect ratio is analysed in this paper. Natural frequencies of a cylinder under uniformly distributed axial loads acting on its bases are calculated numerically by the Ritz method with terms of power series in the coordinate directions as approximating functions. The effect of axial loads on the flexural vibration cannot be described by applying infinitesimal strain theory, therefore, geometrically nonlinear strain-displacement relations with second-order terms are considered here. The natural frequencies of free-free, clamped-clamped, and sliding-sliding cylinders subjected to axial loads are calculated using the proposed three-dimensional Ritz approach and are compared with those obtained with the finite element method and the Bernoulli-Euler theory. Different experiments with cylinders axially compressed by a hydraulic press are carried out and the experimental results for the lowest flexural frequency are compared with the numerical results. An approach based on the Ritz formulation is proposed for the flexural vibration of a cylinder between the platens of the press with constraints varying with the intensity of the compression. The results show that for low compressions the cylinder behaves similarly to a sliding-sliding cylinder, whereas for high compressions the cylinder vibrates as a clamped-clamped one.
\end{abstract}

The subject of wave propagation in rods has been well documented in several classic books [1,2]. Flexural vibrations of long thin cylindrical rods can be described by the basic Bernoulli-Euler (BE) theory of beams. The simplifications introduced make it possible to reduce the three-dimensional problem to a one-dimensional problem. Rayleigh improved beam theory by considering the rotary inertia of cross-sections. Timoshenko beam theory includes shear and rotary effects and provides more satisfactory results for shorter wavelengths.

Exact solutions of the three-dimensional equations of elasticity for an infinite, elastic cylinder were obtained by Pochhammer and Chree in the XIXth century. For finite-length cylinders, there are no general analytical solutions to the equations of motion that fulfil the boundary conditions. Accurate solutions for the natural frequencies of finite-length free cylinders for traction-free end surfaces have been presented by Hutchinson [3,4]. His three-dimensional solution is based on combining exact solutions of the governing equations in series. Heyliger [5] has used the Ritz method with power series

\footnotetext{
* Corresponding author.

E-mail address: felixjose.salazar@upm.es (F.J. Salazar).
} 


\begin{tabular}{|c|c|}
\hline Nomenclature & $\begin{array}{l}\text { non-dimensional axial force (along the } \\
O Z \text { axis) }\end{array}$ \\
\hline cross section area & radial coordinate, non-dimensional radial \\
\hline determined & coordinate \\
\hline experimental data & length-diameter ratio \\
\hline elastic constants matrix & time \\
\hline $\begin{array}{l}\text { d, } A_{i j}, B_{k b}, C_{p q} \text { column vector of coefficients and its } \\
\begin{array}{l}\text { components } \\
\text { diameter of cylinder }\end{array}\end{array}$ & $\begin{array}{l}\mathbf{u}, u, v, w \text { displacement vector and its components } \\
U, V, W \text { displacement functions for the vibration in } r \\
\theta, \text { and } z \text { directions }\end{array}$ \\
\hline initial strain energy per unit volume & $U_{0}, V_{0}, W_{0}$ displacement functions for the static dis- \\
\hline$e_{p}, E_{p} \quad$ strain energy density and strain energy & placement in $r, \theta$, and $z$ directions \\
\hline Young's modulus & axial coordinate, non-dimensional axial \\
\hline kinetic energy & coordinate \\
\hline work done by external forces & strain tensor and its components \\
\hline frequency & a component of strain tensor at initial stage \\
\hline$f_{u}, f_{v}, f_{w} \begin{array}{l}\text { boundary condition functions for the } \\
\text { vibration }\end{array}$ & $\begin{array}{l}\text { strain tensor for vibration and its coefficients } \\
\text { azimuthal coordinate }\end{array}$ \\
\hline $\begin{array}{l}f_{u n^{\prime}}^{\prime}, f^{\prime}{ }_{v n^{\prime}}, f_{w n^{\prime}}^{\prime} \text { boundary condition functions for the } \\
\text { static displacement }\end{array}$ & $\begin{array}{l}\text { Poisson's ratio } \\
\text { density }\end{array}$ \\
\hline axial force and its component along the & stress tensor and its components \\
\hline$O Z$ axis & $\begin{array}{l}\text { stress tensor and its components at } \\
\text { initial stage }\end{array}$ \\
\hline stiffness matrix & angular frequency \\
\hline length of cylinder & non-dimensional frequency \\
\hline mass matrix & $i, j, k, l, p, q$ indices \\
\hline mode order & $I, J, K, L, P, Q$ maximum exponents in series \\
\hline $\begin{array}{l}\text { order of a term in the summation for the } \\
\text { static displacement }\end{array}$ & $\begin{array}{ll}\mathrm{c}-\mathrm{c} & \text { clamped-clamped } \\
\text { sl-sl } & \text { sliding-sliding }\end{array}$ \\
\hline
\end{tabular}

in the coordinate axes directions as the approximating functions to obtain estimates for the axisymmetric free vibrations of finite anisotropic cylinders. Heyliger and Jilari [6] have also studied the free vibration of hollow and orthotropic cylinders of finite length. Visscher et al. [7] have proposed a formulation in rectangular Cartesian coordinates. The Ritz method was also used by Leissa and So $[8,9]$ to study vibrations of isotropic cylinders of finite length that have arbitrary boundary conditions: the three-dimensional components are expressed as algebraic polynomials in the radial and axial directions and as Fourier series in the circumferential direction.

The problem of vibrations of a beam under the action of forces applied on the bases is of interest in civil and mechanical engineering applications. For a BE beam under the axial tensile force, analytical solutions are obtained for the natural frequencies of a simply supported beam [10]. The result shows that natural frequencies increase as the tensile force stiffens the beam while the frequency decreases for the case of a compressed beam. Bokaian [11,12] has studied the influence of both compressive and tensile axial loads on the natural frequencies and mode shapes of beams with various end constraints by solving the equation of motion for flexural waves in thin prestressed rods. His comprehensive study provides an analysis with a quick simple estimate of the natural frequencies of beams under axial loads. He concluded that flexural natural frequencies decrease with the magnitude of compression for free-free and clamped-clamped cylinders and for others types of constraints. Kanaka et al. [13] have also shown that external axial compressive forces reduce the natural frequencies.

Different dynamic methods have been proposed to evaluate axial forces acting on beams and thin rods. The method proposed by Livingston et al. [14] permits the estimation of the tensile force in prismatic beams by using modal data from flexural vibration. A discrete model of an Euler beam elastically supported at its ends is used. Tullini and Laudiero [15] have proposed an analytical approach to evaluating axial forces in slender beams. They solved the eigenvalue problem for a simply supported prismatic beam constrained by two end elastic springs. Amabili et al. [16] have developed a technique for in situ identification of the tensile force in tie-rods in ancient monumental masonry buildings. In these approaches, either the Euler $[14,15]$ or Timoshenko [16] beam is used. The experiments reported refer to slender rods, the ratio between the length and the relevant transverse dimension being larger than approximately 100 . Vibration was generated by an impact hammer and accelerometers were used to detect the vibrations.

Unlike most of the above referred works whose results are applicable to slender bars, a three-dimensional study of the effect of prestressing on the flexural frequencies of a free solid cylinder is performed here. The study includes cylinders with arbitrary length-diameter ratios. The solid cylinder is first subject to axial loads. Then, the natural frequencies of flexural vibration of the axially loaded cylinder about its statically deflected position are calculated. The three-dimensional 
frequency results are obtained by applying the Ritz method with algebraic polynomials in the form of power series in the axial and radial coordinates [8,9]. Terms with exponent zero in $r$ are added to both the radial and tangential series in order to achieve the desired convergence and avoid singularities in calculating the strain energy [17]. Moreover, the Ritz method is optimally applied by following an automatic iterative process that provides the best combination of exponents [18].

The three-dimensional Ritz analysis of the flexural vibration of axially loaded solid cylinders is performed here by applying geometrically nonlinear theory [19] with second-order terms in the strain-displacement relationship. The nonlinear strain is used to take into account the effect of stresses produced by the static axial loads on the flexural vibration. On the other hand, a linear strain-displacement relationship is used to calculate the strain energy due to the small-amplitude flexural vibration superimposed on the axial prestress. Other authors, for instance Pellicano and Amabili [20], have studied the vibration of thin cylindrical shells under axial loads but a different procedure based on Donnell's nonlinear shallow-shell theory is used. Moreover, this analysis is two- dimensional.

The dependence of the natural frequencies on the stress leads us to propose an approach for estimating the axial load to which the cylinder is subjected. Other authors [14-16] have developed this idea, but their proposals are valid for slender bars, whereas a three-dimensional theory is used in the present study.

We also consider constrained cylinders, with both ends clamped or sliding. Numerical results for the flexural natural frequencies obtained by the Ritz method are compared with those obtained by the finite element method (FEM) and by Bernoulli-Euler theory.

Different experiments are carried out in the laboratory, the cylinder is set into free vibration by a transverse impact and the resulting flexural vibration is detected at a point using a laser interferometer. The cylindrical sample is compressed using a hydraulic press and a few natural frequencies for different compressions are measured.

An approach with changing boundary conditions for the displacements, depending on the compression load, is proposed in order to fit the experimental results to the theoretical solution.

\section{Flexural vibration of a circular cylinder}

Let us consider an elastic cylinder of diameter $D$, length $L$, cross-section area $A$, density $\rho$, shear modulus $G$, and Poisson's ratio $v$. A cylindrical coordinate system is placed with its origin at the centre of the cylinder and with the $O Z$-axis aligned with the cylinder axis. The coordinates of a point of the cylinder are $r, \theta$, and $z$ (see Fig. 1 ). The plane of the figure coincides with the plane $y=0$. The components of the displacement $\mathbf{u}$ of a point at the instant $t$ are $u, v$, and $w$ in the radial, circumferential, and axial directions, respectively. The applied force $\mathbf{F}$ is axial in this paper and its $O Z$ component is $F$.

Hamilton's principle for a vibrating system in a natural mode over an oscillation period can be written as

$$
\delta \int_{0}^{2 \pi / \omega} E_{k} \mathrm{~d} t-\delta \int_{0}^{2 \pi / \omega} E_{p} \mathrm{~d} t+\int_{0}^{2 \pi / \omega} \mathrm{d} t \iint_{A} \frac{\mathrm{d} \mathbf{F}}{\mathrm{d} A} \cdot \delta \mathbf{u} \mathrm{d} A=0 .
$$

In this study, potential energy $E_{p}$ refers only to strain energy. The external forces considered to be acting on the system are only the superficial ones. The work, $E_{w}$, done by the external forces $\mathbf{F}$ on the cylinder has not been included in $E_{p}$. The expressions for the kinetic energy $E_{k}$ and the strain energy $E_{p}$ in terms of the linear strain tensor are given by Petyt [21].

The mathematical function for the displacements of a cylinder under axial loads that is vibrating in its flexural modes should include the effect of both the static displacement due to axial loads and that produced by the flexural vibration. The function that relates the displacements to the angle must be periodic in $\theta$. Displacements can therefore be decomposed into a Fourier series. The flexural vibration under investigation is symmetric with respect to the plane $\theta=0$, thus both $u$ and $w$ can be expanded into Fourier cosine series while $v$ is in a sine series. We can consider the harmonic motion or natural mode described by the components of displacement:

$$
u=f_{u}(r, z) U(r, z) \cos (n \theta) \sin (\omega t)+\sum_{n^{\prime}} f_{u n^{\prime}}^{\prime}(r, z) U_{0}(r, z) \cos \left(n^{\prime} \theta\right),
$$

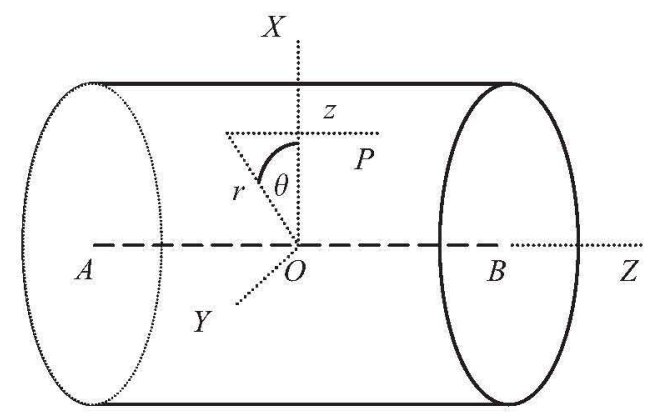

Fig. 1. Cylinder has diameter $D$ and length $L$. Its aspect ratio is $s=L / D$. Coordinates of point $P$ are $r, \theta$, and $z$, and $x, y$, and $z$. 


$$
\begin{gathered}
v=f_{v}(r, z) V(r, z) \sin (n \theta) \sin (\omega t)+\sum_{n^{\prime}} f_{v n^{\prime}}^{\prime}(r, z) V_{0}(r, z) \sin \left(n^{\prime} \theta\right), \\
w=f_{w}(r, z) W(r, z) \cos (n \theta) \sin (\omega t)+\sum_{n^{\prime}} f_{w n^{\prime}}^{\prime}(r, z) W_{0}(r, z) \cos \left(n^{\prime} \theta\right),
\end{gathered}
$$

where components $u, v, w$ must satisfy the boundary conditions for the displacements. Functions $f_{u}, f_{v}, f_{w}, f_{u n^{\prime}}^{\prime}, f_{v n^{\prime}}^{\prime}$, and $f_{w n^{\prime}}^{\prime}$ must guarantee that both initial and boundary conditions are satisfied. $U_{0}, V_{0}$, and $W_{0}$ are previously calculated by applying static principles and these functions correspond to a strain state prior to vibration. The present study refers only to the circumferential flexural mode, $n=1$. Functions $U(r, z), V(r, z)$, and $W(r, z)$ are unknown and must be such that the resulting displacements $u, v, w$ satisfy Hamilton's principle.

To calculate $U(r, z), V(r, z)$, and $W(r, z)$, the Ritz method is applied with powers of coordinates as the basis functions. The series terms have a degree of at least zero in $r$ and the exponents are integers. The strain tensor has terms of the type $u / r$ and $(1 / r)(\hat{\partial} v / \hat{\partial} \theta)$. Therefore, there are some unbounded terms for $r^{0}$. The unbounding problem should be avoided in the calculation of the strain energy. In the study of a free-free non-prestressed rod in flexural vibration [17] it was shown that suitable series are:

$$
\begin{gathered}
U=\sum_{j=0}^{J} A_{0 j} z^{j}+\sum_{i=1, j=0}^{I, J} A_{i j} r^{i} z^{j}, \\
V=-\sum_{j=0}^{J} A_{0 j} z^{j}+\sum_{k=1, l=0}^{K, L} B_{k l} r^{k} z^{l}, \\
W=\sum_{p=1, q=0}^{P, Q} C_{p q} r^{p} z^{q} .
\end{gathered}
$$

Substitution of Eqs. (5)-(7) into (2)-(4) allows $E_{k}, E_{p}$, and $E_{w}$ to be expressed as functions of $D, L, F, A_{i j}$, $B_{k l}$, and $C_{p q}$, the density, and the elastic constants of the material. Eq. (1) allows us to calculate the unknown coefficients $A_{i j}, B_{k l}$, and $C_{p q}$. As Eqs. (5)-(7) are linear in these coefficients, Eq. (1) is expected to lead to a set of linear equations whose compatibility condition permits the calculation of the natural frequencies $\omega$.

For symmetric flexural modes, the displacements of the points of the cylinder are symmetrical with respect to the plane $z=0$, and, therefore, exponent $q$, for the $z$-coordinate in the expression for $W$, only takes odd values whereas exponents $j$ and $l$, in $U$ and $V$, respectively, only take even values. The mode in which the displacement vector is symmetrical to the opposite vector with respect to the plane $z=0$ is known as the antisymmetric mode. In antisymmetric modes $j$ takes odd values and $q$ even values (zero included).

The numerical calculation is considerably simplified by introducing non-dimensional quantities. Non-dimensionalization is accomplished by making use of the non-dimensional coordinates: $r^{\prime} \equiv 2 r / D ; z^{\prime} \equiv z / L$. The slenderness or aspect ratio is defined as $s=L / D$. The non-dimensional frequency is given by $\Omega \equiv \omega D / 2 \sqrt{\rho / G}$. The non-dimensional axial force $N$ (along the $O Z$ axis) is defined as the quotient $N=F /(E A)$, which also represents the static longitudinal relative deformation in a prismatic bar.

A method that provides the optimal exponents [18] in the power series is applied. The optimization method used yields the best (and lowest) value of the lowest non-dimensional frequencies of the first flexural modes by following an automatic iteration process. The optimization method and the improved series are used in the numerical calculations described in some of the following sections in the study of the flexural vibration of a cylinder under axial loads.

\section{Axially prestressed cylinders: linear theory}

Let us consider the natural state of the cylinder (without stress and in repose). Stress is first applied and the initial state (in repose) is reached. Prestressing forces are caused by compressive or tensile forces uniformly distributed on the end surfaces of the cylinder. Later the cylinder is set into flexural vibration by a transverse impact. For the prestressed free-free cylinder vibrating in its flexural modes, no geometric boundary conditions are imposed on the displacements $\left(f_{u}, f_{\nu}, f_{w}, f_{u n^{\prime}}^{\prime}, f_{v n^{\prime}}^{\prime}, f_{w n^{\prime}}^{\prime}\right.$ disappear from (2)-(4)) and the total displacements can be expressed in the form:

$$
\begin{gathered}
u=U(r, z) \cos \theta \sin (\omega t)+U_{0}(r, z), \\
v=V(r, z) \sin \theta \sin (\omega t)+V_{0}(r, z), \\
w=W(r, z) \cos \theta \sin (\omega t)+W_{0}(r, z),
\end{gathered}
$$

where

$$
\begin{gathered}
U_{0}(r, z)=-v N r, \\
V_{0}(r, z)=0,
\end{gathered}
$$




$$
W_{0}(r, z)=N z
$$

The conditions for unbounding to be prevented are the same as those considered for free vibration of a non-prestressed cylinder [17]. Thus, Eqs. (5)-(7) can also be applied.

The derivatives of expressions (8)-(10) with respect time do not depend on $N$, therefore neither does the kinetic energy depend on $N$. Substitution of Eqs. (5)-(7) into (8)-(10) and, then, the resulting displacements into the formula for strain energy [21] (with linear strain-displacement relations) yields that $N$ appears only in the form of $-v N$, in both terms $u / r$ and $\partial u / \partial r$, and as $N$ in the terms corresponding to $\partial w / \partial z$. By calculating the squares in the expression of $E_{p}$, it is found that some terms have the common factor $N \sin (\omega t)$, others the factor $N^{2}$, and others $\sin ^{2}(\omega t)$. When integrating strain energy over a period of time, the results obtained show that the integral with the first factor becomes zero, the integral with the second factor does not depend on unknown coefficients, and the integral with the third factor is independent of $N$. Thus, in calculating the variation of the action due to potential energy, resulting from the variation of the coefficients, the final expression does not depend on $N$.

The variation of the action due to the external axial forces at the end $z=L / 2$ (or $z=-L / 2$ ) is

$$
\iint_{A} \frac{\mathrm{d} \mathbf{F}}{\mathrm{d} A} \cdot \delta \mathbf{u d} A=\frac{F}{A} \iint_{A} \delta W(r, L / 2) \cos \theta \sin (\omega t) r \mathrm{~d} r \mathrm{~d} \theta=0 .
$$

Therefore, it can be concluded that, for this model and with the assumptions made, flexural natural frequencies do not depend on the axial load applied on the cylinder. Numerical calculation by the Ritz method confirms this result.

\section{Axially prestressed cylinders: geometrically nonlinear strain}

It is assumed that the constitutive relations of the material follow Hooke's law, stress is then proportional to strain; i.e. the material is linear elastic. Fig. 2 qualitatively represents one of the components of the stress tensor $\tau$ in terms of one of the components of the strain tensor $\boldsymbol{\varepsilon}$. The cylinder acquires the initial strain $\varepsilon_{i}$ by gradually applying the stress from zero to $\tau_{i}$. It is assumed that the area $e_{\mathrm{pi}}$ of the triangle provides a part of the initial strain energy per unit volume. When the cylinder is set into flexural vibration, the strain increases by $\varepsilon_{v}$ at a certain instant $t$, which results in an increase of the strain energy density that is equal to the area of the trapezium formed by the rectangle with area $e_{p}^{\prime}$ plus the triangle with area $e_{p}^{\prime \prime}$. Furthermore, the term $e_{p}$ of the strain energy per unit volume can be considered as the sum of the areas of the triangles $e_{\mathrm{pi}}$ and $e_{p}^{\prime \prime}$ plus the area of the rectangle $e_{p}^{\prime}: e_{p}=e_{\mathrm{p} 1}+e_{p}^{\prime}+e_{p}^{\prime \prime}$. In the following calculations, the strain energy of the system in the initial state is chosen as the reference energy, therefore $e_{p}$ can be written simply as:

$$
e_{p}=e_{p}^{\prime}+e_{p}^{\prime \prime}
$$

In what follows in this section, the displacements $u, v$, and $w$ are determined by the terms varying with time in Eqs. (8)-(10).

The cylinder under axial external forces vibrates freely in the flexural modes, but the displacements due to the vibration are so small that a linear strain-displacement relation for the strain $\boldsymbol{\varepsilon}_{\mathrm{v}}$ is assumed to be sufficient to calculate the strain energy associated with the vibration given by the upper triangle in Fig. 2. Then, the strain energy $E_{p}^{\prime \prime}$ of the whole cylinder

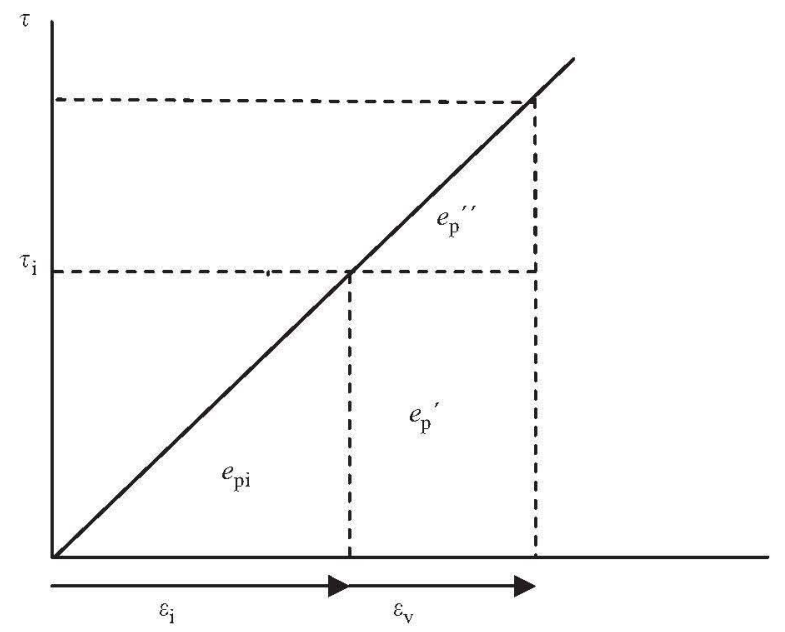

Fig. 2. Graph of a component of stress tensor against a component of strain tensor for an elastically linear material. Area of triangle with base $\varepsilon_{1}$ gives initial strain energy, $e_{\mathrm{pi}}$. When flexural vibration is induced, strain increases by $\varepsilon_{v}$ at a given instant $t$. 
can be expressed in the form:

$$
E_{p}^{\prime \prime}=\frac{1}{2} \int_{V o l} \varepsilon_{\mathrm{V}}^{\mathrm{T}} \mathbf{C}_{\mathrm{V}} \mathrm{d} V o l,
$$

where $\mathbf{C}$ is the elastic constants matrix and the vector $\boldsymbol{\varepsilon}_{\mathrm{v}}$ is computed using linear strain theory. The potential $E_{p}^{\prime \prime}$ is calculated by the customary procedures [21].

Novozhilov [19] indicated the limitations of infinitesimal strain theory for solving problems such as that of bending complicated by the presence of axial loads. In order to calculate the term $e_{p}^{\prime}$ of strain energy density for the rectangle, a nonlinear strain approximation was used $[22,23]$ to define the components of the strain tensor. Thus, in this case quadratic terms are included in the components of the strain tensor for the flexural vibration, $\boldsymbol{\varepsilon}_{\mathrm{v}}$. Therefore, the strain energy $E_{p}^{\prime}$ expressed in matrix form is given by:

$$
E_{p}^{\prime}=\int_{V o l} \tau_{i}^{T} \varepsilon_{\mathrm{v}} \mathrm{d} V o l
$$

where $\tau_{1}$ is the initial stress vector (six independent components) that exits in the cylinder prior to the vibration process and the superscript $T$ denotes the transpose. The column vector $\varepsilon_{v}=\left[\varepsilon_{r r, v} ; \varepsilon_{\theta \theta, v} ; \varepsilon_{z z, v} ; \varepsilon_{r \theta, v} ; \varepsilon_{r z, v} ; \quad \varepsilon_{\theta z, v}\right]$ corresponds to the six independent strain components in cylindrical coordinates. These components are calculated using nonlinear strain theory $[19,24]$.

For the axially prestressed cylinder at the initial stage, all the components of the stress tensor are equal to zero except for $\tau_{z z, i}=F / A$, which is correct because it satisfies the equilibrium conditions and the boundary conditions. Therefore, according to the rectangle shown in Fig. 2, $E_{p}^{\prime}$ can be written as:

$$
E_{p}^{\prime}=\int_{V o l} \frac{F}{A} \varepsilon_{z z, v} \mathrm{~d} V o l=\frac{F}{A} \int_{V o l}\left[\frac{\partial w}{\partial z}+\frac{1}{2}\left(\frac{\partial u}{\partial z}\right)^{2}+\frac{1}{2}\left(\frac{\partial v}{\partial z}\right)^{2}+\frac{1}{2}\left(\frac{\partial w}{\partial z}\right)^{2}\right]_{v} \mathrm{~d} V o l
$$

where the component $\varepsilon_{z z, v}$ of nonlinear strain tensor in cylindrical coordinates has been obtained from known considerations $[19,24]$.

When the flexural displacements of Eqs. (8)-(10) are substituted into (18), it is found that the integral of the first term in the integrand in (18) is zero as result of the integral of $\cos \theta$ on the interval $(0,2 \pi)$. The quadratic terms cause the integral to not be zero. It is concluded that the nonlinear terms (quadratic terms) in the strain component $\varepsilon_{z z, v}$ should be included in Eq. (18) in order to detect the presence of axial forces acting on a cylinder vibrating in the flexural modes.

Natural flexural frequencies of a cylinder under axial loads are calculated in this study by using Hamilton's principle (1). As already demonstrated, the variation of the action due to the work of the axial forces is zero, thus Hamilton's principle is reduced to

$$
\delta \int_{0}^{2 \pi / \omega} E_{k} \mathrm{~d} t-\delta \int_{0}^{2 \pi / \omega} E_{p} \mathrm{~d} t=\delta \int_{0}^{2 \pi / \omega} E_{k} \mathrm{~d} t-\delta \int_{0}^{2 \pi / \omega} E_{p}^{\prime} \mathrm{d} t-\delta \int_{0}^{2 \pi / \omega} E_{p}^{\prime \prime} \mathrm{d} t=0 .
$$

Let us apply the Ritz method and analyze the variation of the action of the terms in Eq. (19). The displacement functions $U, V, W(5)-(7)$ are a linear function of the coefficients $A_{i j}, B_{k l}, C_{p q}$, as are the displacements $u, v, w$ given by (8)-(10). Substitution of the displacements (8)-(10) into the expression for kinetic energy [21] yields:

a) Kinetic energy is a quadratic function of the coefficients and, consequently, variation of the action due to kinetic energy is a linear function of the coefficients.

b) Kinetic energy produces the common factor $\omega^{2} \sin ^{2}(\omega t)$ and its action over a period gives $\omega$ as a common factor.

In the same way, substitution of the displacements for the vibration into Eq. (16) for strain energy $E_{p}^{\prime \prime}$ allows us to conclude that:

c) Since in this equation a linear strain-displacement relation is used, $E_{p}^{\prime \prime}$ is a linear function of the products of two coefficients and, therefore, the variation of the action related to this strain energy is a linear function of the coefficients.

d) The action corresponding to this strain energy produces the common factor $\cos ^{2}(\omega t)$ and its action over a period, the factor $1 / \omega$.

Finally, the variation of the action of Eq. (18) yields that the corresponding term in (19) is a linear function of the coefficients and the common factor $1 / \omega$ is also obtained.

Thus, the application of Hamilton's principle is simplified and the resulting set of equations is linear, which can be expressed after some manipulation in the usual matrix form:

$$
\mathbf{K d}-\Omega^{2} \mathbf{M d}=\mathbf{0},
$$

where $\mathbf{d}$ is the column matrix of the coefficients and $\mathbf{K}$ and $\mathbf{M}$ are the stiffness and mass matrices, respectively. It should be noted that matrix K includes the effect of axial loads. Non-dimensional frequency $\Omega$ is a function of $s, v$, and $N$, i.e. $\Omega=\Omega(s$, $v, N)$. Natural frequencies can therefore be calculated by solving a standard eigenvalue problem. 
Table 1

Values of lowest flexural natural frequency $f_{1}$ in $\mathrm{Hz}$ in terms of axial force applied $F$, or non-dimensional force $N=F /(E A)$, for a free-free $c y l i n d e r$ made of stainless steel, with aspect ratio $s=10$, diameter $D=50 \mathrm{~mm}$, Poisson's ratio $v=0.298$, and Young's modulus $E=198 \mathrm{GPa}$. Frequencies are calculated by optimized Ritz method (column RI), by using BE theory (column BE), and by FEM analysis (column FE). The quotient of the two lowest frequencies is also shown.

\begin{tabular}{|c|c|c|c|c|c|}
\hline$F(\mathrm{MN})$ & $N$ & RI & $f_{2} / f_{1} \mathrm{RI}$ & $\mathrm{BE}$ & $\mathrm{FE}$ \\
\hline-3 & -0.00772 & - & - & - & - \\
\hline-2.3 & -0.00592 & 119.8 & 33.00 & 183.2 & 130.3 \\
\hline-2.2 & -0.00566 & 218.0 & 18.20 & 260.8 & 223.6 \\
\hline-2.1 & -0.00540 & 283.9 & 14.02 & 319.2 & 288 \\
\hline-2 & -0.00514 & 337.1 & 11.86 & 370.8 & 340.2 \\
\hline-1.5 & -0.00386 & 526.6 & 7.725 & 552 & 528 \\
\hline-1 & -0.00257 & 662.6 & 6.244 & 686.8 & 663.1 \\
\hline-0.8 & -0.00206 & 709.3 & 5.871 & 733.6 & 709.6 \\
\hline-0.6 & -0.00154 & 752.9 & 5.566 & 777.2 & 753.1 \\
\hline-0.4 & -0.00103 & 794.0 & 5.312 & 818.4 & 794 \\
\hline-0.2 & -0.00051 & 832.9 & 5.096 & 857.6 & 832.8 \\
\hline 0 & 0 & 870.0 & 4.911 & 894.8 & 869.7870 .1 \\
\hline 0.2 & 0.00051 & 905.3 & 4.748 & 930.4 & 904.9 \\
\hline 0.4 & 0.00103 & 939.2 & 4.605 & 964.8 & 938.8 \\
\hline 0.6 & 0.00154 & 971.8 & 4.477 & 997.6 & 971.3 \\
\hline 0.8 & 0.00206 & 1003 & 4.362 & 1030 & 1002.6 \\
\hline 1 & 0.00257 & 1034 & 4.259 & 1060 & 1032.9 \\
\hline
\end{tabular}

\section{Flexural natural frequencies of free-free cylinders subjected to axial loads by the Ritz method}

The procedure described in Section 4 is applied to determine the free vibration frequencies of solid prestressed cylinders. The Ritz method with the improved series (5-7) and the optimization procedure described in [18] are used in order to calculate accurate values of the natural flexural frequencies. Table 1 shows the results for a free-free cylinder made of stainless steel with $v=0.298, E=198 \mathrm{GPa}, D=50.00 \mathrm{~mm}$, and $s=L / D=10$. A convergence study is made for the first frequency, beginning with a small value of the number of coefficients and increasing such a number until the desired convergence is achieved. The lowest non-dimensional frequency converges to four digits when 40 coefficients are used.

The first column of Table 1 shows the force applied $F$, in the second the non-dimensional force $N$, and in the third (column titled RI) the lowest frequency $f_{1}$ for symmetric modes calculated by the Ritz method. The quotient $f_{2} / f_{1}=\Omega_{2} / \Omega_{1}$ is also tabulated in Table $1\left(f_{2} / f_{1} \mathrm{RI}\right)$.

From Table 1 it can be deduced that for the cylinder analysed it is sufficient to measure the lowest symmetric flexural frequency and then select the value of the frequency measured in the column RI. The columns for $F$ and $N$ then provide the values of the force in $\mathrm{MN}$ and the non-dimensional force, respectively.

Thus, if for any cylinder, without restrictions on the displacements in the bases, data $L, D, \rho, G$, and $v$ are known, in principle, it is sufficient to excite and measure the lowest flexural natural frequency and to calculate a table similar to Table 1 in order to be able to estimate the axial stress to which the cylinder is subjected.

The solid line in Fig. 3 (obtained from Table 1) shows the variation of the lowest flexural frequency, in Herz, of the stainless steel cylinder being studied with the non-dimensional axial force. It should be noted that maximum variation is observed for stresses close to the compressive stress that would produce buckling. Thus, the sensitivity of the method for the calculation of the stress from the lowest flexural frequency is also at a maximum for those values of compression.

Fig. 3 (dashed line) also shows the quotient $f_{2} / f_{1}$ as a function of $N$, also plotted from the data shown in Table 1 (slenderness $s=10$ ). Fig. 3 shows a great variation in the quotient of the two lowest frequencies against the applied force, particularly for large compressive forces. From Fig. 3 it can be inferred that the ratio of the two lowest symmetrical flexural frequencies of a free-free cylinder also allows us to estimate the stress $N$ applied and, therefore, to calculate $F$ provided that the area of the cylinder and its Young's modulus are known. As the absolute value of the slope is at a maximum in the region close to buckling, the sensitivity of the calculation will be at a maximum in that region.

The table of values of $\Omega_{1}$ in terms of $N$ for a short cylinder is also calculated by the Ritz method using the nonlinear approximation. Calculations are performed for the symmetric modes and for different values of the force acting on the bases of a cylinder with dimensions $L=D=50.00 \mathrm{~mm}(s=1), v=0.298, E=198 \mathrm{GPa}(\mathrm{G}=76.3 \mathrm{GPa})$, and density $\rho=7884 \mathrm{~kg} / \mathrm{m}^{3}$. The Ritz method uses 95 terms and the optimized exponents are (7, 8, 5, 8, 6, 9). The solid line in Fig. 4 shows the result obtained for the lowest flexural frequency for theoretical relative deformation smaller than $8 \%$. Fig. 4 shows an important variation of the frequency, although the interval of stress studied is very wide in order to show the tendency to buckling. From the numerical data for $\Omega_{1}$ and $\Omega_{2}$, the variation of the quotient of frequencies, $f_{2} / f_{1}$, with the stress is also calculated. The results obtained show that for this short cylinder such a variation is not significant even though the values of $N$ are large. Moreover, the quotient of frequencies increases with the magnitude of stress in a short cylinder but decreases in a cylinder with $s=10$. Hence, calculation of the axial stress by means of the quotient of frequencies seems to be not applicable to short cylinders. 


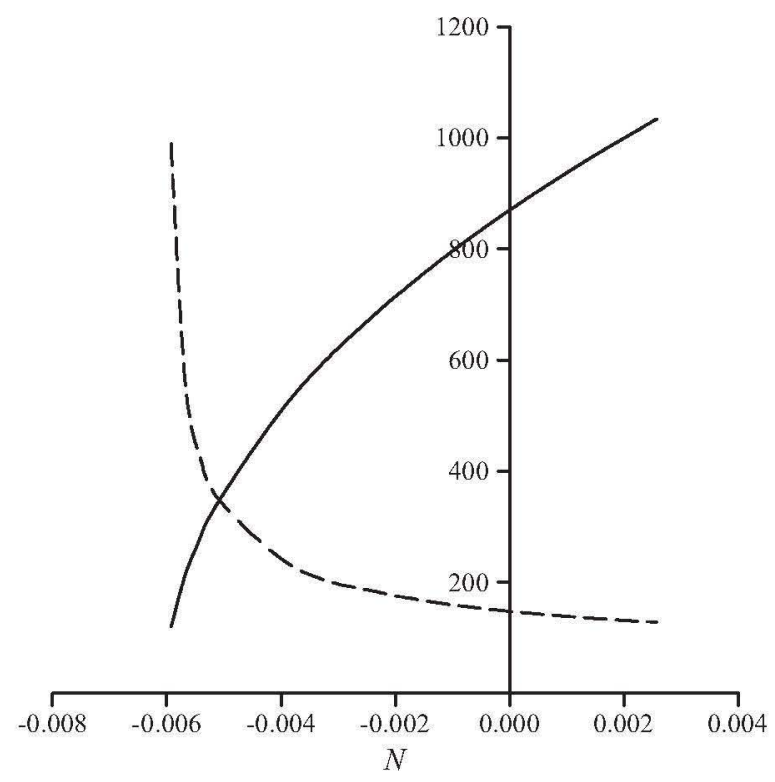

Fig. 3. Variations of lowest flexural frequency $f_{1}$ and quotient $f_{2} / f_{1}$ with non-dimensional force $N$ for a free-free steel cylinder of $L=10 D$ by Ritz method. Solid line represents $f_{1}$ in $\mathrm{Hz}$. Dashed line denotes quotient of two lowest frequencies $f_{2} / f_{1}$ for symmetric modes and vertical axis represents the product of 30 and this quotient $\left(30 \times f_{2} / f_{1}\right)$. Values of $f_{1}$ and $f_{2} / f_{1}$ as a function of $N$ seem to be good candidates for calculating stress from lowest flexural natural frequencies.

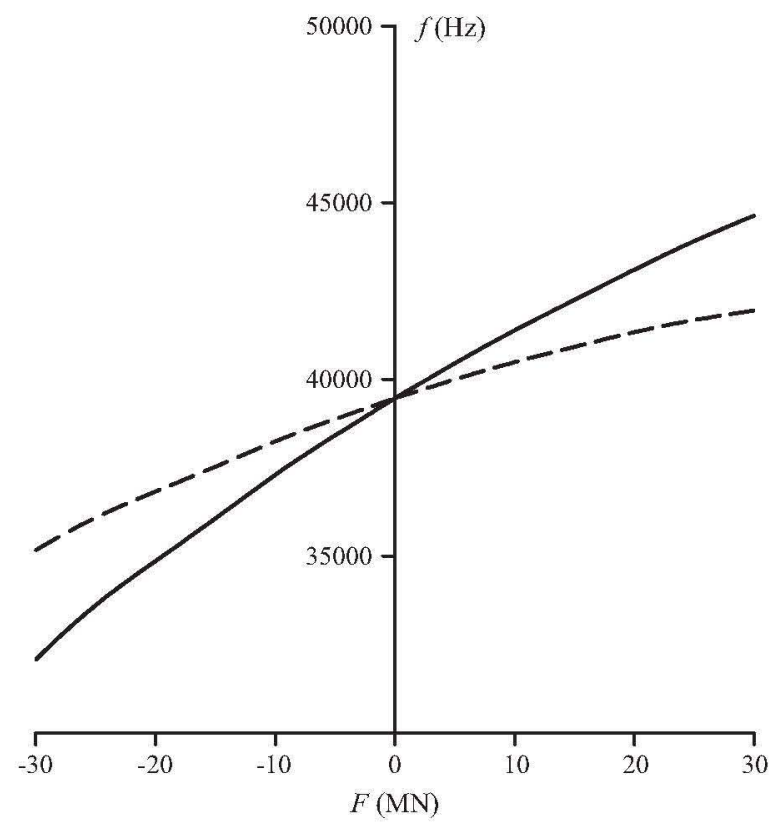

Fig. 4. Flexural vibration frequency of a free-free steel cylinder of $L=D=50.00 \mathrm{~mm}$ versus applied force $F$. Solid line corresponds to Ritz method and dashed line to FEM.

The fifth column of Table 1 also gives the frequencies calculated from the solution of the differential equation given by beam theory BE $[11,12]$.

\section{Natural frequencies of prestressed cylinders by FEM analysis}

For the purpose of comparing the Ritz results with the three-dimensional solution obtained by FEM analysis, the preceding calculations are repeated using the commercial program Ansys ${ }^{\circledR}$, for the stainless cylinders, $E=198 \mathrm{GPa}$, $v=0.298, \rho=7884 \mathrm{~kg} / \mathrm{m}^{3}$. The cylinders vibrate freely without any constraint. 


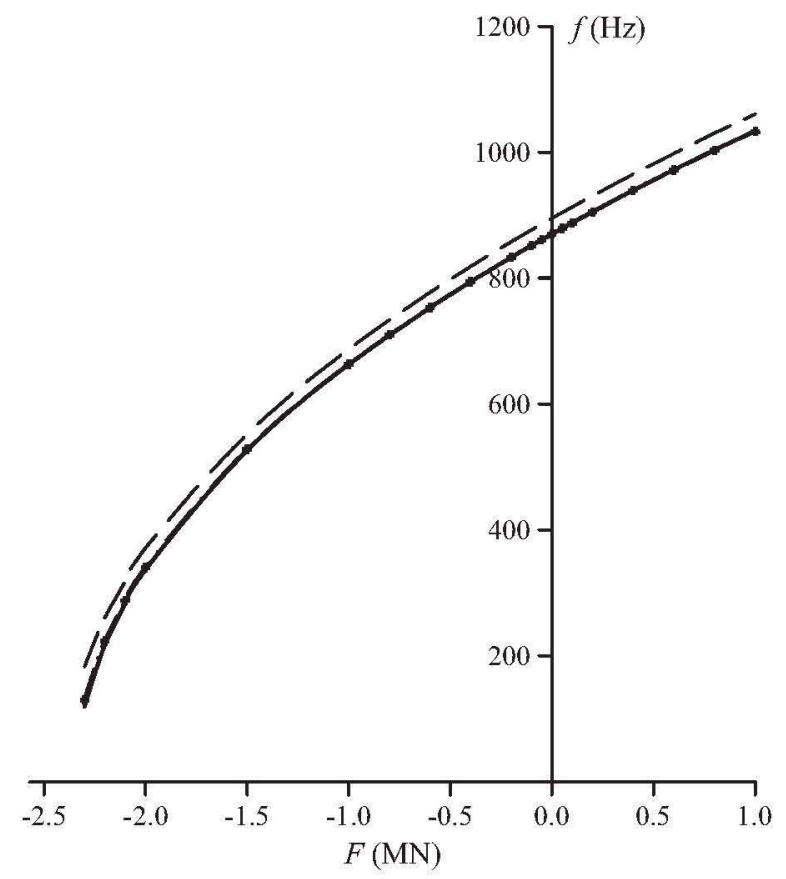

Fig. 5. Variation of lowest flexural vibration frequency with applied force for a free-free steel cylinder of $s=10, D=50.00 \mathrm{~mm}$, obtained by BE theory (long dashed line), by FEM (squares), and by Ritz procedure (solid line).

In order to include the effect of the axial forces acting on both ends, the total force is divided by the number of nodes at the ends and the resulting value is applied to each node. Two models are considered in the simulation performed: the first is static and the other is dynamic (modal). The results obtained with the static model are transferred to the dynamic one so that the calculated effects of prestressing are taken into account in the modal analysis. The procedure is essentially the same as a regular modal analysis, except that the cylinder is first prestressed (static analysis). The effect of stress stiffening is accounted for by generating and then using an additional stiffness matrix, which is added to the regular stiffness matrix in order to give the total stiffness.

A study of the sensitivity of the model to the mesh size was performed before the simulation was undertaken. The mesh was refined until the variation in the frequency in two sequential simulations was negligible. The element dimensions are $D / 30$ in $X$ and $Y$ directions and $L / 200$ in $Z$ direction. The number of nodes is 166,629 .

Calculations for a cylinder with $L=10 \mathrm{D}$ were performed in order to minimize the effect of the distribution of forces (Saint Venant). The results for the prestressed and free-free cylinder are shown in Table 1 . The values of the frequency by FEM are listed in the sixth column (titled FE).

Fig. 5 shows the results of Table 1. The long dashed line corresponds to the BE theory applied by Bokaian [11,12], the solid line to the results by the Ritz method, and the squares to FEM. The FEM and Ritz results are in good agreement. It should be noted that the corresponding lines are superimposed.

Calculations were repeated for the free-free short cylinder with $L=D=0.050 \mathrm{~m}$. A table of frequencies was calculated for the different values of the force applied. Fig. 4 represents the results: the solid line corresponds to the Ritz method and the dashed line to the FEM results. The discrepancies between the frequencies calculated by the Ritz method and FEM procedures are not significant for values of the force included in the interval $\pm 1 \mathrm{MN}$. However, the results are not in as much agreement as in the case of the more slender cylinder $(L=10 D)$ for the high values of the force shown in Fig. 4 , corresponding to large theoretical compressive and tensile forces up to $30 \mathrm{MN}$. It should be noted that the variations of the frequency with the prestressing are more significant in the results obtained by the Ritz method than in those calculated by FEM analysis.

\section{Experimental results with $F=0$}

In order to experimentally verify the numerical results, the numerically calculated flexural natural frequencies were compared with the experimentally measured frequencies. The sample chosen for the experiments was the most complicated one for numerical calculations, the cylinder with an aspect ratio $s=1$. This sample can be considered to be neither a slender cylinder nor a disc, thus the simple theories commonly applied do not provide accurate results. However, for the experiment to be simple, the particular case of $F=0$ was considered. The sample is a stainless steel cylinder with $L=D=49.90 \mathrm{~mm}, \rho=7884 \mathrm{~kg} / \mathrm{m}^{3}, v=0.298$, and $G=76.3 \mathrm{GPa}$. The cylinder was placed with its axis in the horizontal 


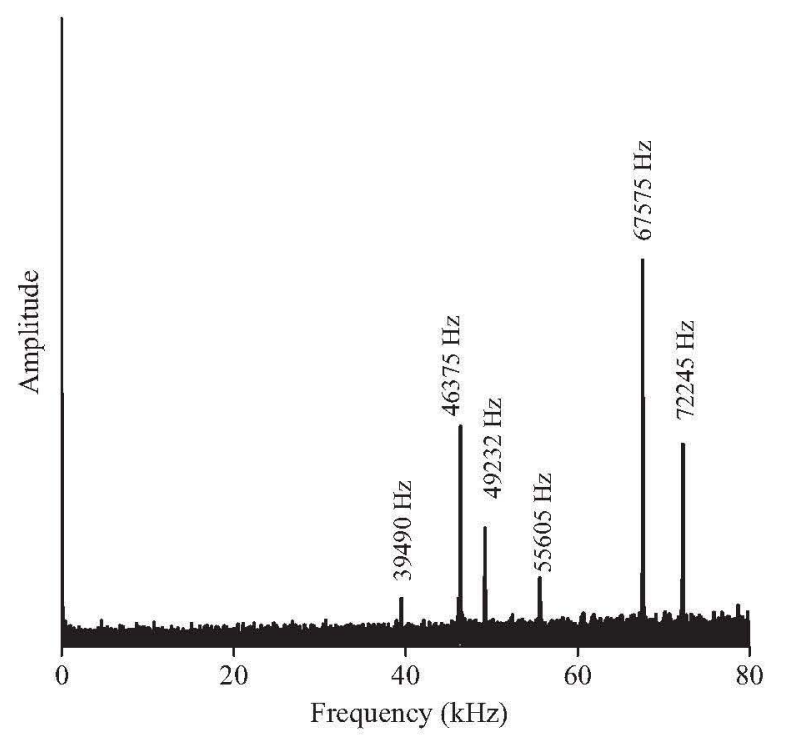

Fig. 6. Spectrum of free flexural vibration of a steel cylinder with $L=D=49.90 \mathrm{~mm}$.

position, supported on two rubber blocks so that it could vibrate almost freely. A small steel ball held by a thread formed a simple pendulum, which was used to apply a horizontal impact, perpendicular to the axis of the cylinder and at the centre of the cylindrical surface of the sample. Thus, a broad frequency spectrum in the flexural symmetrical modes is excited and the cylinder vibrated almost freely after the brief impact. The out-of-plane velocity component at a point on the lateral cylindrical surface was detected by a laser Doppler vibrometer (Polytec OFV-5000) with a VD-06 velocity decoder. The laser interferometer had a frequency range of $0-350 \mathrm{kHz}$ and a velocity range of $0-2 \mathrm{mms}^{-1} / \mathrm{V}$. Surface preparation was not required. A small area was illuminated by the laser beam (point-like detection) so that the detection point on the cylindrical surface was diametrally opposite the impact point. The signal detected by the laser interferometer was digitalized by an oscilloscope (Tektronik TDS5032B) to calculate its fast Fourier transform (FFT). Maxima in the FFT spectrum correspond to the natural vibration frequencies of the cylinder tested. Fig. 6 shows the FFT spectrum obtained in this experiment. The lowest experimental natural frequency was $f_{1 \mathrm{e}}=39,490 \mathrm{~Hz}$.

\section{Comparison of results}

a) Comparison of BE solution with the Ritz three-dimensional numerical results:

As has already been mentioned, BE beam theory [11-12] for $s=10$ gives the results given in Table 1 (column $\mathrm{BE}$ ). Frequencies calculated by the Ritz procedure ( 40 coefficients) are also listed in Table 1, column RI. Comparing the values of the frequencies given in these columns, a considerable discrepancy is obtained (BE theory always gives larger values for the frequencies), the difference being approximately 3\%. Fig. 5 shows the results.

b) Comparison of So's results with those calculated by the Ritz optimized method with $F=0$ :

The repetition of the calculation of the lowest frequency for $L=D$ and $v=0.3$ gives $\Omega_{1}=1.99380$ for $F=0$ and 85 coefficients. By applying So's proposal [25], he obtained the same value with 126 coefficients. The fact that the value of the frequency obtained here by the optimized method is equal to that calculated by So with more coefficients, shows that the procedure used here really improves the calculation of the flexural frequency.

c) Comparison of results from Ritz method and FEM analysis:

From the results obtained in Sections 5 and 6 for the cylinder studied with $s=10$ (see Table 1), it can be deduced that the values calculated for the lowest flexural frequency by applying the Ritz method and FEM analysis are in good agreement. When the cylinder is under tensile or compression prestressing with non-dimensional forces (relative deformations) of up to 0.0026 , the differences between the Ritz solution and the FEM results are not greater than $0.1 \%$. In the compression zone, the differences are greater than $1 \%$ for $F$ larger than $2 \mathrm{MN}$. That is to say, there is only disagreement between the results for the frequencies in the zone of large relative deformations, corresponding to loads close to the theoretical value that would cause buckling.

For the cylinder with $s=1$, the differences in the values of the flexural frequency, calculated by both methods, are more significant (see Fig. 4). For apparent deformations smaller than $0.26 \%$, the differences between the values of frequencies calculated by the Ritz and FEM procedures are smaller than $0.23 \%$. However, such a difference increases considerably with the initial stress but it does not exceed $1.2 \%$ until the non-dimensional force reaches a value of 0.013 . For compressive loads and a theoretical relative deformation of $7.7 \%$ the frequencies differ by up to $9.7 \%$. 
d) Comparison of results obtained by the Ritz method for $F=0$ with experimental results:

The lowest flexural frequency that was measured experimentally was $f_{1 \mathrm{e}}=39,490 \mathrm{~Hz}$ whereas for the same cylinder the numerical calculation by the Ritz method yielded a value of $f_{1 R}=39,549 \mathrm{~Hz}$. Then, the values differ by $-0.15 \%$. This agreement demonstrates that both the numerical calculation of the natural frequency by means of the optimized Ritz method with the improved series and the experimental measurement are very accurate.

Calculation of the second and the third frequencies gives $55,553 \mathrm{~Hz}$ and $67,448 \mathrm{~Hz}$, respectively. The relative differences of these values with respect to the experimental values given in Fig. 6, 55,605 Hz and 67,575 Hz, are $0.09 \%$ and $0.19 \%$, respectively. From tables calculated by So [25] for $v=0.3$ and the rest of properties equal to those of our stainless steel sample, it is deduced that the frequencies $46,375 \mathrm{~Hz}$ and $49,232 \mathrm{~Hz}$ correspond to the two lowest modes with $n=2$, and the frequency $72,245 \mathrm{~Hz}$ corresponds to $n=3$ in Eqs. ((2)-(4)).

\section{Prestrained and clamped-clamped cylinder}

Consider a cylinder placed between the platens of a press in the compression experiment. The platens of the press are assumed to be flat, parallel, and rigid surfaces. Both the rigid platens and the end surfaces of the cylinder are also assumed to be very well polished, the reason why the coefficient of friction between both materials is small. The force that applies the press increases slowly bringing both platens closer. The friction force is small at this first stage. Thus, Hooke's historical experiment is being repeated. Let $F$ be the final applied force (negative for compression).

Once the cylinder is compressed, flexural vibration is set up in the cylinder by a percussion perpendicular to its axis. If the friction between the cylinder and the press is now assumed to be high due to the high value of $F$, the points on both bases cannot move with respect to the platens. For this reason, it is assumed that the bases of the cylinder are clamped to the platens of the press during the flexural vibration, i.e. the vibration displacement components $u, v$, and $w$ are zero at $z= \pm L / 2$. The proposed displacements for the clamped-clamped cylinder (c-c model) are:

$$
\begin{gathered}
u=\left(z^{2} / L^{2}-1 / 4\right) U(r, z) \cos \theta \sin (\omega t)+U_{0}(r, z), \\
v=\left(z^{2} / L^{2}-1 / 4\right) V(r, z) \sin \theta \sin (\omega t)+V_{0}(r, z), \\
w=\left(z^{2} / L^{2}-1 / 4\right) W(r, z) \cos \theta \sin (\omega t)+W_{0}(r, z) .
\end{gathered}
$$

The model of vibration proposed by Eqs. (21)-(23) fulfils the initial conditions since at $t=0$ the resulting displacement represents the state of the compressed cylinder. This proposal also satisfies the boundary conditions for the displacements at both ends, $z= \pm L / 2$. Eqs. (21)-(23) represent at any time a harmonic motion of each point about its equilibrium position when the cylinder is under the static axial load prior to the vibrating stage. The factor $z^{2} / L^{2}-1 / 4$ is even in $z$, therefore the parity or symmetry of the vibration depends on the parity of $U(r, z), V(r, z)$, and $W(r, z)$.

The conditions for strain energy to be finite are demonstrated to be the same as those for the free-free cylinder and, therefore, the series for $U, V$, and $W$ are those given by Eqs. (5)-(7).

The virtual work done by the forces that the fixed platens apply to the bases during the vibration is zero. Therefore, Eq. (19) can also be applied to the clamped-clamped cylinder but the displacements used in the Ritz method are those given by Eqs. (21)-(23). In order to apply Eq. (19), kinetic energy $E_{k}$ and strain energies $E_{p}^{\prime}$ and $E_{p}^{\prime \prime}$ are calculated, as explained in Section 4 . It is only necessary to consider the displacements due to the vibration for the calculation of the strain energy $E_{p}{ }^{\prime}$. Thus, the second terms in (21)-(23) are not involved in the calculation of $E_{p}^{\prime}$. Analogously, these terms do not contribute to the variation of the action due to $E_{p}^{\prime \prime}$.

Table 2 shows some of the results calculated for the stainless steel cylinder with the following properties: $D=50.00 \mathrm{~mm}, s=10, \rho=7884 \mathrm{~kg} / \mathrm{m}^{3}, v=0.298$, and clamped-clamped ends. The first and second columns give the force and non-dimensional force, respectively. Numerical calculation by the optimized Ritz method with 41 coefficients shows that the lowest frequency corresponds to a symmetric mode and the results appear in the third column. The fourth column shows the values of the frequencies calculated with the BE approach. The values of the frequencies calculated by FEM analysis are listed in the fifth column (titled FECC).

Fig. 7 displays some of the results shown in Table 2. For $F=0$ the values calculated by FEM analysis and the Ritz method (column titled RICC) agree, except for a $0.7 \%$ difference. For relative deformations of $0.26 \%$ corresponding to tensile forces, the values calculated by the optimized Ritz method and FEM analysis differ by $0.43 \%$. If this deformation is caused by compression, then the difference between the frequencies obtained by the Ritz method and FEM analysis is $1.1 \%$. As regards the discrepancies in applying $\mathrm{BE}$ theory and the Ritz procedure, the differences obtained for such a relative deformation are $3.5 \%$ and $4 \%$ for tensile and compressive loads, respectively. The difference between the values of the lowest flexural frequency calculated by the Ritz and FEM procedures for compressed cylinders does not exceed $5 \%$ until forces greater than $-5 \mathrm{MN}$ are applied (relative theoretical deformation of $1.3 \%$ ). For values of the force close to the theoretical critical buckling force, the differences between the Ritz and FEM solutions increase moderately but the results by BE and RICC differ significantly. 
Table 2

Lowest flexural natural frequency $f_{1}$ in $\mathrm{Hz}$ as a function of $F$ and $N$ for a clamped-clamped cylinder of stainless steel, aspect ratio $s=10$, and $D=50 \mathrm{~mm}$. This frequency is calculated by the following methods: Ritz (RICC), BE theory (BECC), and FEM analysis (FECC). Results obtained with a sliding-sliding model by means of Ritz method are also included (RISS).

\begin{tabular}{|c|c|c|c|c|c|}
\hline$F(\mathrm{MN})$ & $N$ & $\operatorname{RICC} f(\mathrm{~Hz})$ & $\operatorname{BECC} f(\mathrm{~Hz})$ & $\operatorname{FECC} f(\mathrm{~Hz})$ & $\operatorname{RISS} f(\mathrm{~Hz})$ \\
\hline-9.5 & -0.0244 & - & 89.2 & - & - \\
\hline-9.3 & -0.0239 & - & 158.8 & - & - \\
\hline-9.2 & -0.0237 & - & 184.8 & - & - \\
\hline-9 & -0.0232 & - & 226 & - & - \\
\hline-8.9 & -0.0229 & - & 244.4 & - & - \\
\hline-8.8 & -0.0227 & 81.7 & 261.2 & 234.3 & - \\
\hline-8.7 & -0.0224 & 124.4 & 277.2 & 251.4 & - \\
\hline-8.6 & -0.0222 & 155.8 & 292.4 & 267.5 & - \\
\hline-8.5 & -0.0219 & 181.8 & 306.8 & 282.5 & - \\
\hline-8 & -82.519 & 277 & 370 & 348.1 & - \\
\hline-7 & -0.018 & 403.9 & 470.8 & 450.5 & - \\
\hline-6 & -0.0155 & 498.4 & 553.2 & 532.8 & - \\
\hline-5 & -0.0129 & 576.8 & 624.4 & 603.2 & - \\
\hline-4 & -0.0103 & 645 & 687.6 & 665.6 & - \\
\hline-3 & -0.00773 & 706 & 745.2 & 722.1 & - \\
\hline-2 & -0.00515 & 761.7 & 799.2 & 774 & 148.9 \\
\hline-1 & -0.00258 & 813.1 & 848 & 822.3 & 294.6 \\
\hline 0 & 0 & 861.1 & 894.8 & 867.5 & 389.1 \\
\hline 1 & 0.00258 & 906.3 & 938.8 & 910.2 & 464.7 \\
\hline 2 & 0.00515 & 949 & 980.8 & 950.7 & 529.7 \\
\hline 3 & 0.00773 & 989.6 & 1021 & 989.2 & 587.5 \\
\hline 4 & 0.0103 & 1028 & 1059 & 1026 & 640.1 \\
\hline 5 & 0.0129 & 1065 & 1096 & 1061 & 688.7 \\
\hline 8 & 0.0206 & 1169 & 1199 & 1160 & 817.4 \\
\hline
\end{tabular}

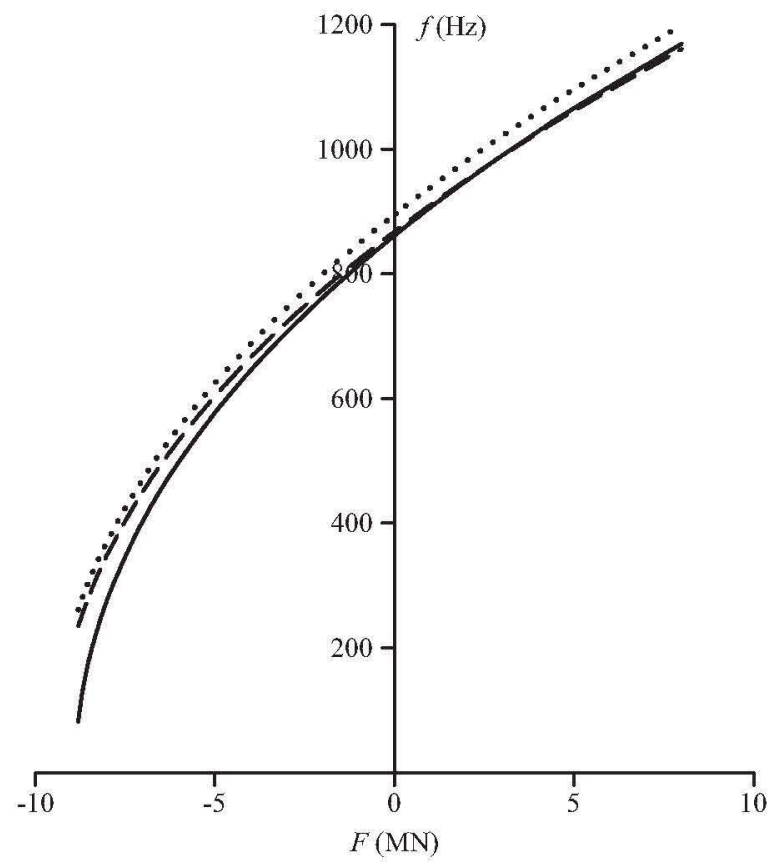

Fig. 7. Flexural vibration frequency for a cylinder of $L=10 \mathrm{D}$ with clamped-clamped ends. Results obtained applying BE theory (dotted line), FEM analysis (dashed line), and Ritz method (solid line).

\section{Prestrained and sliding-sliding cylinder}

Another interesting case is that of a cylinder whose bases are forced to remain in contact with the platens of a press without friction forces so that the points of the bases of the cylinder can move freely in the plane of the platens. However, movement towards the platens is prevented (unilateral constraints). A model is proposed in which the displacements of all the points of the cylinder can move freely, apart from the out-of-plane component of the displacement of the points on the 


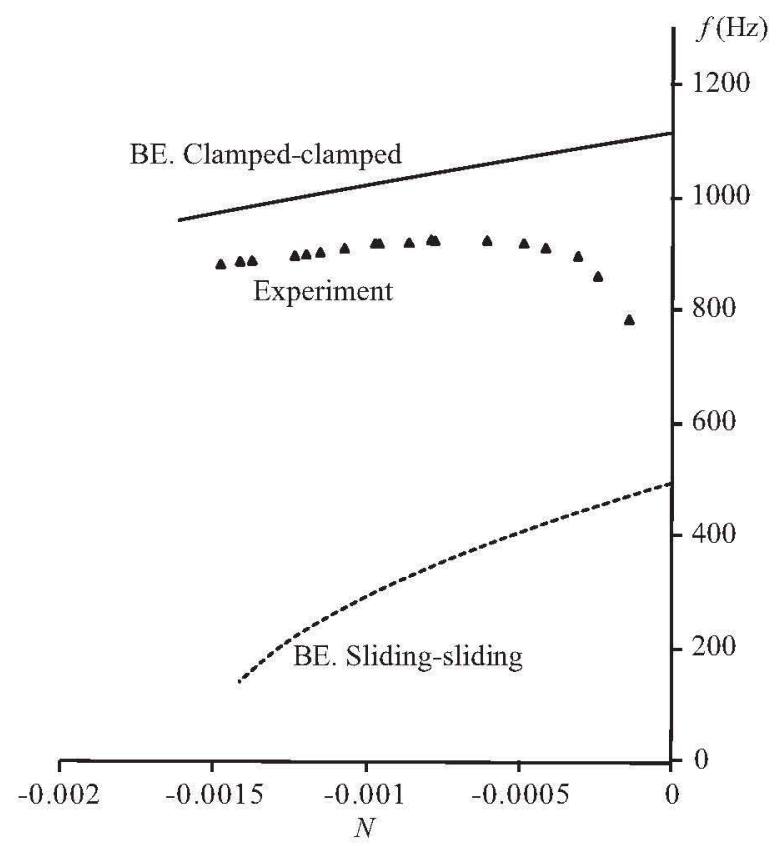

Fig. 8. Theoretical results (BE) and experimental measurements for lowest flexural natural frequency against $N$ for a steel cylinder with a diameter of $10 \mathrm{~mm}$ and a length of $200 \mathrm{~mm}$.

bases that is zero (bilateral constraint), i.e. sliding-sliding conditions (sl-sl model). Therefore, the displacement component $w$ due to the flexural vibration must be zero at $z= \pm L / 2$. Then, the displacement components can be written as follows:

$$
\begin{gathered}
u=U(r, z) \cos \theta \sin (\omega t)+U_{0}(r, z), \\
v=V(r, z) \sin \theta \sin (\omega t)+V_{0}(r, z), \\
w=\left(z^{2} / L^{2}-1 / 4\right) W(r, z) \cos \theta \sin (\omega t)+W_{0}(r, z) .
\end{gathered}
$$

This model will not exactly represent the process of compression with a press since the condition that $w=0$ at both ends can be very difficult to satisfy. Moreover, it is also difficult to satisfy the freedom of movement for $u$ and $v$ in an experiment with friction between the press and the bases of the cylinder.

The application of the procedure described in Section 4 and the optimized Ritz method with 41 coefficients to the cylinder with $s=10$ yields the values of the lowest frequency in term of the force applied (see column in Table 2 (titled RISS)). The great difference between the values that appear in this column and in the others is due to the fact that the lowest flexural frequency for the sliding-sliding boundary conditions corresponds to an antisymmetric mode, whereas for the clamped-clamped case it corresponds to a symmetric mode.

For a steel cylinder with $s=20$, Fig. 8 shows the values of the frequency in terms of the non-dimensional force, using BE theory [11], which was used because the calculations are very simple. The curves are for the clamped-clamped model (solid line) and for the sliding-sliding model (dashed line). Fig. 8 shows a decrease in the value of the frequency as the compression increases; the decrease is more noticeable in the sl-sl model since the critical compression load is smaller.

\section{Experimental results with a hydraulic press}

The hydraulic press with which the experiments were carried out was a table press, provided with a manual handle for the injection of the liquid into the piston, see Fig. 9. The press was equipped with a dynamometer graduated in $\mathrm{kN}$ and with a sensitivity of $0.1 \mathrm{kN}$. The platens were of steel and their masses were much greater than that of the cylinders tested. The cylinder is supported by the two platens of the press. The force applied increased gradually up to a maximum value, trying not to approach the buckling limit. At each step of the compression process, vibration was induced as described in Section 7. The experiments were carried out with slender cylinders ( $s=10$ and 20 ). The results obtained were:

a) Stainless steel cylinder with $L=200.00 \mathrm{~mm}, D=10.00 \mathrm{~mm}, s=20.00, \rho=7884 \mathrm{~kg} / \mathrm{m}^{3}, E=198 \mathrm{GPa}$, and $v=0.298$. The lowest flexural frequency measured was $f_{1}=1087 \mathrm{~Hz}$ for the freely vibrating cylinder. Deformations of $0.2 \%$ were not reached in these experiments. 


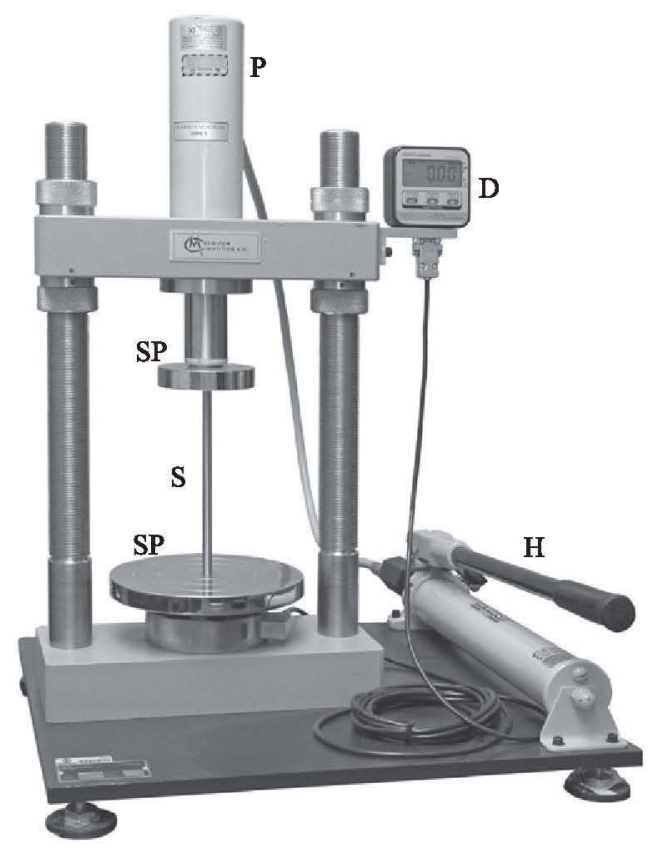

Fig. 9. Experimental setup showing the press and the cylindrical sample: steel cylinder (S); steel platens (SP); manual handle (H) for the injection of the liquid into the piston (P); dynamometer (D) graduated in $\mathrm{kN}$.

Table 3 gives the experimental results of the flexural frequencies for this steel cylinder. The triangles of Fig. 8 graphically show the results of Table 3, i.e. the variation of the values of the frequency measured with the applied force. Fig. 8 shows that there is:

a1) A sharp increase in the value of the measured frequency with the compression, followed by a maximum, and finally a slow decrease in the frequency.

a2) The values of the experimental frequencies are between those corresponding to sliding-sliding and clampedclamped ends.

a3) There is an almost linear decrease for high compressions. The experimental frequencies seem to tend towards the theoretical values for clamped-clamped ends.

The square of the lowest frequency as a function of the applied force, for both the theoretical solution given by BE theory and the experimental measurements, is shown in Fig. 10. It should be noted that, for high compressions, the relationship between the square of the experimentally measured frequencies and the force is almost linear. The linear fit of these values for high compressions gives a square of the correlation coefficient of 0.9956; a value close to the exact value of 1 , which was obtained by applying BE theory. This result shows a good correlation with the experimental data in the zone of high compressions.

b) Commercial brass cylinder of $L=15.49 \mathrm{~cm}, D=1.50 \mathrm{~cm}, \rho=8417 \mathrm{~kg} / \mathrm{m}^{3}, E=98.4 \mathrm{GPa}, G=36.4 \mathrm{GPa}$.

A table was built from the values of the experimentally measured frequencies in terms of the force applied to the brass bar. The experimental values are represented by crosses in Fig. 11. Unlike the steel bar, a maximum in the plot of the frequency versus force is not reached. For that reason a second experiment was carried out with the brass bar in order to extend the interval of $N$ under study, the range also included small forces. Fig. 11 shows the results of both experiments, triangles represent the values corresponding to the second experiment. The line drawn between the crosses and triangles is obtained as an average of the frequencies measured in both experiments for the interval common of forces. The two lines corresponding to $\mathrm{BE}$ theory are also shown. The same comments can be made about Fig. 11 as were made about Fig. 8.

In the second experiment deformations greater than $0.3 \%$ were reached. Plastic deformation occurred for values above $60 \mathrm{kN}$. It seems that the interval of interest, corresponding to a dependence of the frequency on the force obtained theoretically for a clamped-clamped bar, is in the range $26.22-49.02 \mathrm{kN}$. It should be noted that the values of the frequency increase from $49.02 \mathrm{kN}$ and then an increase in the compressive force leads to plastic deformation.

Extrapolating the linear fit (the square of the frequency versus $N$ ) of the experimental data for the zone of high compressions in the first experiment to estimate the value of the frequency for $N=0$ yields a theoretical frequency for the clamped-clamped cylinder under no prestressing of $f_{1}=1732 \mathrm{~Hz}$. The natural frequency measured is $1795 \mathrm{~Hz}$ when the cylinder, free at both ends, is not prestressed. According to BE theory this value is the same as that corresponding to a clamped-clamped cylinder. The difference between these values is as small as $3.5 \%$. 
Table 3

Values of lowest flexural natural frequency $f_{1}$, experimentally obtained for a stainless steel cylinder of $s=20.00$ and $D=10.00 \mathrm{~mm}$. Cylinder was compressed by a hydraulic press.

\begin{tabular}{cll}
\hline$F(\mathrm{kN})$ & $N$ & $f(\mathrm{~Hz})$ \\
\hline-2.2 & -0.00014 & 782 \\
-3.78 & -0.00024 & 861 \\
-4.78 & -0.00031 & 898 \\
-6.42 & -0.00041 & 913 \\
-7.52 & -0.00048 & 921 \\
-9.38 & -0.00060 & 926 \\
-12 & -0.00077 & 926 \\
-12.2 & -0.00079 & 928 \\
-13.3 & -0.00086 & 923 \\
-14.9 & -0.00096 & 921 \\
-15.1 & -0.00097 & 921 \\
-16.6 & -0.00107 & 913 \\
-17.8 & -0.00115 & 905 \\
-18.5 & -0.00119 & 901 \\
-19.1 & -0.00123 & 899 \\
-21.3 & -0.00137 & 890 \\
-21.9 & -0.00141 & 889 \\
-22.9 & -0.00147 & 884 \\
\hline
\end{tabular}

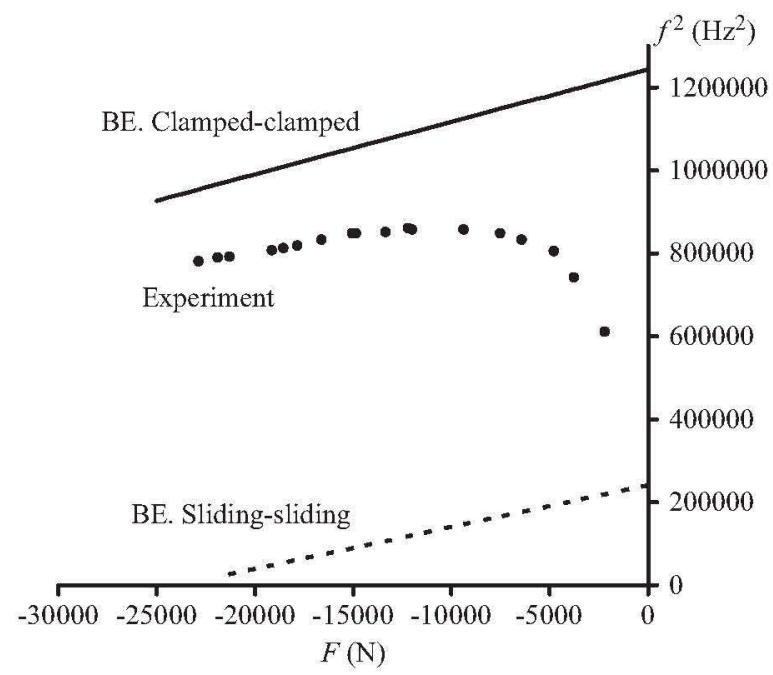

Fig. 10. Square of frequency $f$ as a function of force $F$ for stainless steel cylinder with aspect ratio $s=20$. Results obtained by BE theory for both clampedclamped and sliding-sliding cylinders are two straight lines $(r=1)$. Fit of experimental results for $|F|>17.8 \mathrm{kN}$ to a straight line gives a correlation coefficient of $r=0.9978$.

c) Commercial aluminium cylinder with $L=155.00 \mathrm{~mm}, D=15.00 \mathrm{~mm}, \rho=2840 \mathrm{~kg} / \mathrm{m}^{3}, E=71 \mathrm{GPa}$, and $v=0.33$.

The flexural natural frequencies measured in two experiments carried out with the above-described cylinder are shown in Fig. 12. It should be noted that from a value of the force, which supposedly corresponds to the clampedclamped cylinder, the values obtained in both experiments are similar. Nevertheless, the frequencies measured for low compressions seem to be highly dependent on the boundary conditions, which appear to be different in the two experiments. Fig. 12 also shows the theoretical frequencies calculated by BE theory. The behaviour of the experimental frequency-force curve for aluminium is similar to that of the brass and steel cylinders. From the fitting of the experimental data for the "linear" zone in the plot off ${ }^{2}$ versus $\mathrm{N}$, a theoretical frequency of $f=2449 \mathrm{~Hz}$ is obtained for $F=0$. The value measured when the cylinder is not prestressed is $2715 \mathrm{~Hz}$, both values differ by $11.7 \%$.

Comparing the zones of high compression in Figs. 8 and 11 with those in Fig. 12, it is found that in the latter, corresponding to aluminium, the agreement between the theoretical values for the $\mathrm{c}-\mathrm{c}$ model and the experimental ones 


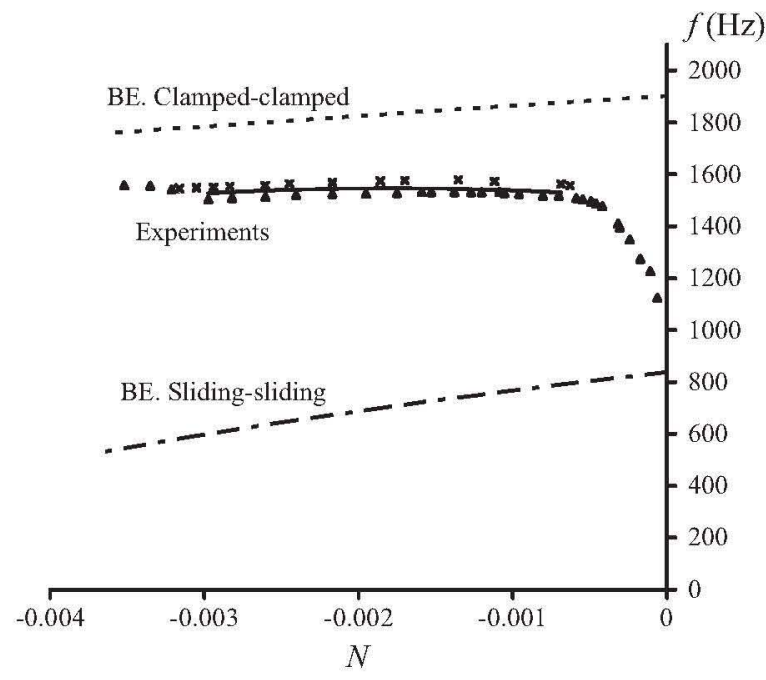

Fig. 11. Flexural natural frequencies for brass cylinder of $s=10.3$ versus compression force applied. Crosses and triangles represent experimental results in first and second series of experiments with same cylinder, respectively. Solid line corresponds to fit of results of both series of measurements for values of compression ranging from $-11.28 \mathrm{kN}$ to $-49 \mathrm{kN}$. Upper and lower lines show frequencies calculated by $\mathrm{BE}$ theory for clamped-clamped and slidingsliding models, respectively.

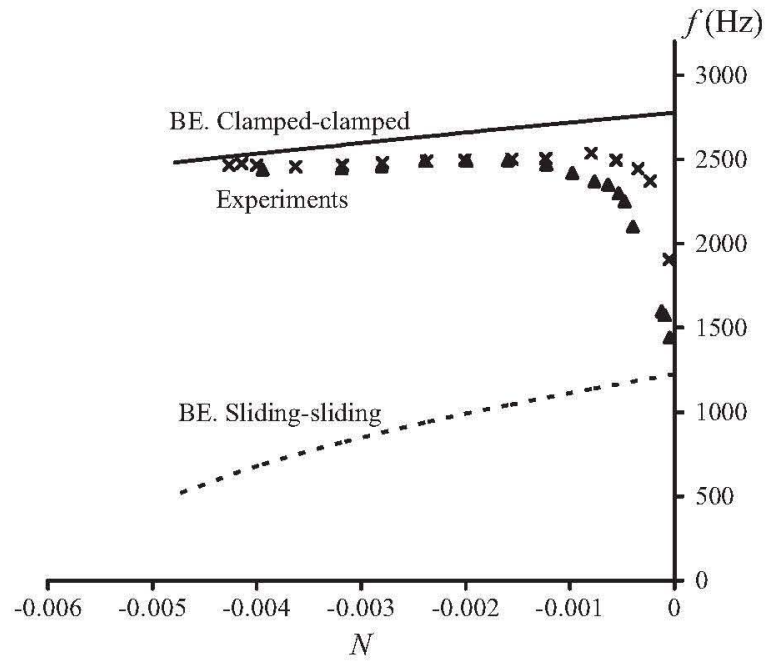

Fig. 12. Flexural natural frequencies of an aluminium cylinder under compression. Triangles represent experimental results for lowest flexural frequency in terms of $N$ and crosses show experimental values in a second experiment. Upper and lower lines show frequencies calculated by BE theory for clamped-clamped and sliding-sliding models, respectively.

is almost perfect. This can be attributed to the fact that aluminium is much softer than both stainless steel and brass. Thus, the relative error made by assuming that the platens are rigid in the $\mathrm{c}-\mathrm{c}$ model is smaller for the aluminium cylinder.

\section{Sliding-sliding/clamped-clamped model}

In view of the results obtained for the three bars tested, it is deduced that, for small compressions, the cylinders behave similarly to the sliding-sliding model whereas for high compressions the behaviour is analogous to that of the clampedclamped model. When the cylinder is subjected to large compression loads, the friction between the loading platens and the two end surfaces constrain the ends from lateral displacements. It seems opportune to return to Section 9 and to consider the boundary conditions depending on the value of the compression. A mixed model for the boundary conditions is proposed below in which the sliding-sliding model and the clamped-clamped one are used for low and high 


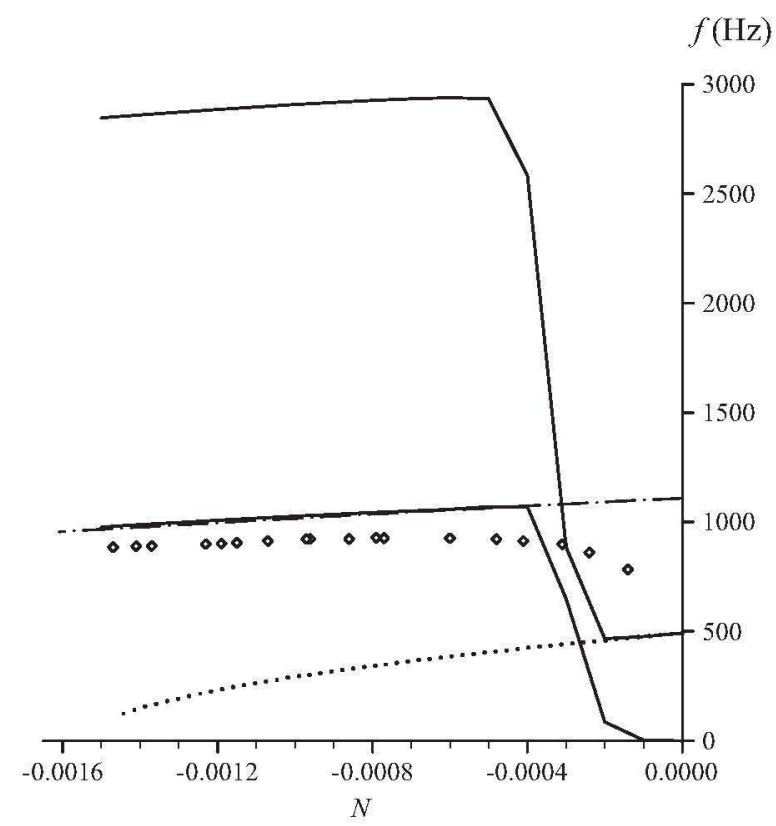

Fig. 13. Ritz method calculation for the mixed model with sliding-sliding/clamped-clamped conditions, for steel cylinder of $s=20$, yields results for first antisymmetric and symmetric modes, shown with upper and lower solid lines, respectively. Experimental results (rhombuses) and values calculated by $\mathrm{BE}$ model for sl-sl and $c-c$ end conditions are also shown in dotted line and dashed-dotted line, respectively.

compressions, respectively. The displacement $w$ is of the form given by Eqs. (23) or (26) because it is assumed that the points of the cylinder located on the end surfaces are in permanent contact with the platens of the press.

In order to introduce an analytical expression which is easy to handle, in the model proposed for the boundary conditions, the displacements depend on the compression $N$ in such a way that there is a smooth transition from sl-sl to c-c conditions. A proposal is:

$$
\begin{aligned}
& u=\left[\frac{1}{4}+\frac{z^{2}}{L^{2}} \tanh (b N)\right] U(r, z) \cos \theta \sin (\omega t)+U_{0}(r, z) \\
& v=\left[\frac{1}{4}+\frac{z^{2}}{L^{2}} \tanh (b N)\right] V(r, z) \sin \theta \sin (\omega t)+V_{0}(r, z)
\end{aligned}
$$

where the non-dimensional parameter $b$ is a constant characteristic of the experiment carried out.

Boundary conditions given by Eqs. (27) and (28) describe the constraints on the radial and tangential displacements induced by compression. The degree of constraint on the radial and tangential displacements on the end surfaces depends on the non-dimensional force $N$. For $N=0$, the expression enclosed in the square brackets in (27) and (28) equals $1 / 4$, the in-plane constraint does not exist, and the ends are free to move in the radial and tangential directions, therefore the resulting displacements represent the sliding-sliding conditions. On the other hand, for large compression loads, the radial and tangential displacements on the end surfaces are completely constrained; indeed, if $N$ tends to $-\infty$, the displacements given by (27) and (28) become those of a clamped-clamped cylinder. For intermediate compressive loads, partial slip can occur between the cylinder and the loading platens.

Friction, as a source of an irreversible process, is not considered here. The dependence of displacements on force $N$, expressed by the square brackets in (27) and (28), can be interpreted as due to the fact that a portion of the bases is clamped due to friction and the rest is free, and the fraction clamped increases with $N$.

The lowest flexural frequencies for the steel cylinder as a function of the compressive force are calculated by the Ritz method for the symmetric modes and then for the antisymmetric ones. The procedure described in Section 4 is followed. The results obtained are shown in Fig. 13 for $b=3000$. The upper and lower solid lines correspond to the antisymmetric and symmetric modes, respectively. It should be noted that the curves for the symmetric and antisymmetric modes do not cross each other. This figure has little detail because the interval between two values of $N$ is of the order of $\Delta N=0.0001$, therefore, the curves are constituted by noticeably straight line segments. The null frequency, corresponding to the motion of the cylinder as a rigid body, is not included. These null frequencies are possible in the sliding-sliding model. The values of the frequency for the sl-sl and $\mathrm{c}-\mathrm{c}$ models (both calculated by the BE theory) are also shown in Fig. 13 along with the experimental results of Table 3 . 


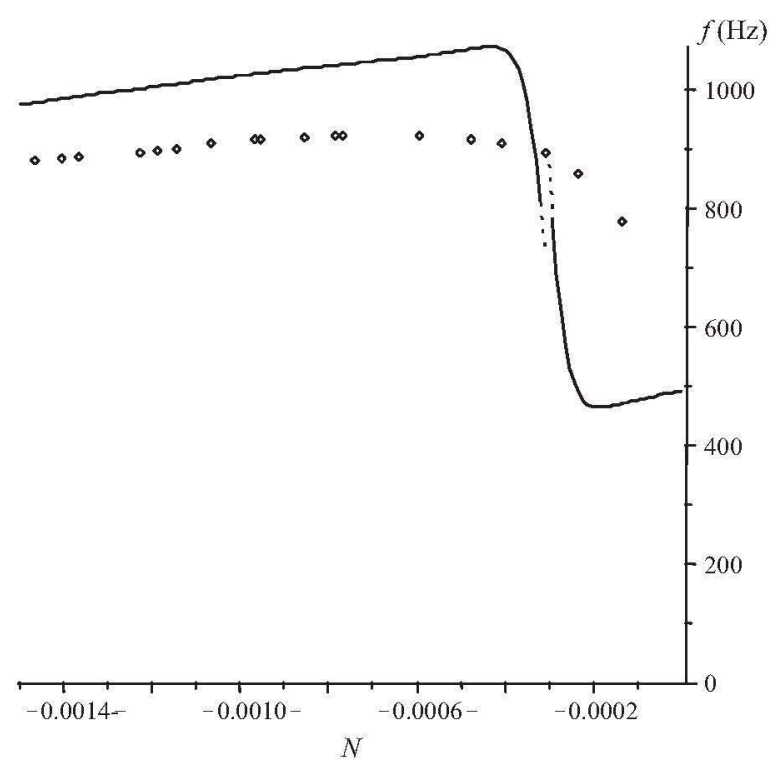

Fig. 14. Curves of variation of lowest flexural natural frequency with non-dimensional compressive force applied to stainless steel cylinder of $s=20$, calculated by Ritz method. Solid double curve qualitatively resembles experimental results denoted by rhombuses.

For the steel cylinder with $s=20$, the curves obtained for the variation of the lowest flexural frequency in terms of the compression applied, with $\Delta N=0.00001$ and $b=3000$, are shown in Fig. 14. The resulting curve resembles qualitatively that showing the experimental results denoted by rhombuses. In order to draw such final curves the following considerations are taken into account:

1) Frequencies close to zero are disregarded in the experiment since they do not indicate vibration and, in addition, can be caused by background noise; therefore they are not listed in Table 3. For that reason the values of almost null frequency in Fig. 13 are disregarded in the final result shown in Fig. 14.

2) For small values of compression, the lowest frequencies should be chosen from the antisymmetric mode, whose smallest value is approximately $500 \mathrm{~Hz}$.

3) For high values of compression, the non-null lowest frequencies correspond to the symmetric mode, and this mode is selected.

4) The lowest frequency measured in the experiment was $782 \mathrm{~Hz}$ and its variation was followed with the gradual increase in compression.

5) There is a region of intermediate values of $N$ in which the curves corresponding to the symmetric and antisymmetric modes almost touch each other; in this region the experimental results show that there are two close frequencies, almost multiple frequencies, as would be expected according to the model.

The curve obtained with these considerations (see Fig. 14) is composed of the two solid curve portions. It bears a qualitatively resemblace to the experimental curve. However, it seems evident that this result could be improved with a more refined curve fitting.

\section{Conclusions}

In this work, the Ritz method is used to investigate the effect of stress on the natural flexural frequencies of a cylinder of any aspect ratio. Three-dimensional solutions are obtained for flexural natural frequencies of cylinders under axial loads. Suitable power series are used to represent the displacements and all the exponents are optimally chosen by following an iterative process. This yields a faster convergence to accurate results for natural frequencies.

A numerical model based on the Ritz method is developed for an axially loaded cylinder. Elastic linear behaviour of the material is assumed but nonlinear strain components are included in the analysis. Nonlinear terms in the strain components associated with relative elongations of elements, which before deformation are parallel to the direction of the axial force, account for the effect of the force on the flexural vibration. On the other hand, a linear strain tensor is considered in evaluating the strain energy associated with the small-amplitude flexural vibration superimposed on its initial tension. 
Natural flexural frequencies of symmetric modes of free-free prestressed cylinders are numerically calculated by applying the Ritz approach. For a free-free cylinder of stainless steel, with aspect ratio 10 and a Poisson's ratio of 0.3 , under compressive forces, and relative strain of $0.2 \%$, a decrease in the lowest frequency of approximately $18 \%$ is observed, while for an aspect ratio of 1 , the decrease is about $0.4 \%$. The approach proposed can be used to evaluate the prestressing force acting on a cylinder from the measurement of the lowest natural frequency.

Laboratory experiments are carried out using a hydraulic press to compress the cylindrical samples. Free flexural vibration is excited by striking the sample with a pendulum and the induced vibration is detected with a laser interferometer. A disagreement is found between the experimental values of the lowest flexural frequency and those calculated by the Ritz method for clamped-clamped and sliding-sliding cylinders. As the compressive force increased, the boundary conditions seemed to change from sliding-sliding conditions to clamped-clamped ends. For high compressive forces, the sample behaves as if it were a clamped-clamped cylinder. A model with changing boundary conditions which is based on the Ritz formulation is proposed. The approach allows for the possibility of considering intermediate cases between full and zero transverse constraint at the ends. The flexural frequencies calculated in terms of the compressive force are in partial agreement with the measurements in the laboratory tests. The discrepancies observed may have been due to factors such as: the nonrealistic modeling of the boundary conditions in the actual compression test, the nonuniform stress distribution within the cylinder under compression due to the friction existing between the end surfaces and the platens of the loading machine, the loss of mechanical energy due to friction, and the uncertainty in the measurement of the applied force.

\section{References}

[1] K.F. Graff, Wave Motion in Elastic Solids, Dover, New York, 1975.

[2] J.D. Achenbach, Wave Propagation in Elastic Solids, North-Holland, Amsterdam, 1993.

[3] J.R. Hutchinson, Vibrations of solid cylinders, Journal of Applied Mechanics 47 (1980) 901-907.

[4] J.R. Hutchinson, Transverse vibrations of beams, exact versus approximate solutions, Journal of Applied Mechanics 48 (1981) 923-928.

[5] P.R. Heyliger, Axisymmetric free Vibrations of finite anisotropic cylinders, Journal of Sound and Vibration 148 (1991) 507-520.

[6] P.R. Heyliger, A. Jilari, The free vibrations of inhomogeneous elastic cylinders and spheres, International Journal of Solids and Structures 29 (1992) $2689-2708$.

[7] W.M. Visscher, A. Migliori, T.M. Bell, R.A. Reinert, On the normal modes of free vibration of inhomogeneous and anisotropic elastic objects, Journal of the Acoustical Society of America 90 (1991) 2154-2162.

[8] A.W. Leissa, J. So, Comparisons of vibration frequencies for rods and beams from one-dimensional and three-dimensional analyses, Journal of the Acoustical Society of America 98 (1995) 2122-2135.

[9] A.W. Leissa, J. So, Accurate vibration frequencies of circular cylinders from three-dimensional analysis, Journal of the Acoustical Society of America 98 (1995) 2136-2141.

[10] W. Weaver, S.P. Timoshenko, D.H. Young, Vibrations Problems in Engineering, John Wiley \& Sons, New York, 1990.

[11] A. Bokaian, Natural frequencies of beams under compressive axial loads, Journal of Sound and Vibration 126 (1988) 49-65.

[12] A. Bokaian, Natural frequencies of beams under tensile axial loads, Journal of Sound and Vibration 142 (1990) 481-498.

[13] K. Kanaka Raju, G. Venkateswara Rao, Free vibration behaviour of prestressed beams, Journal of Structural Engineering 112 (1986) $433-436$.

[14] T. Livingston, J.G. Béliveau, D.R. Huston, Estimation of axial load in prismatic members using flexural vibrations, Journal of Sound and Vibration 179 (5) (1995) 899-908.

[15] N. Tullini, F. Laudiero, Dynamic identification of beam axial loads using one flexural mode shape, Journal of Sound and Vibration 318 (2008) $131-147$.

[16] M. Amabili, S. Carra, L. Collini, R. Garziera, A. Panno, Estimation of tensile force in tie-rods using a frequency-based identification method, Journal of Sound and Vibration 329 (2010) 2057-2067.

[17] A. Bayón, F. Gascón, R. Medina, F.J. Nieves, F. Salazar, Study of pure transverse motion in free cylinders and plates in flexural vibration by Ritz's method, European Journal of Mechanics A/Solids 30 (2011) 423-431.

[18] F.J. Nieves, A. Bayón, F. Gascón, Optimization of the Ritz method to calculate axisymmetric natural vibration frequencies of cylinders, Journal of Sound and Vibration 311 (2008) 588-596.

[19] V.V. Novozhilov, Foundation of the Nonlinear Theory of Elasticity, Dover, New York, 1999, p. 54

[20] Pellicano, Amabili, Stability and vibration of empty and fluid-filled circular cylindrical shell under static and periodic axial loads, International Journal of Solids and Structures 40 (2003) 3229-3251.

[21] M. Petyt, Introduction to Finite Element Vibration Analysis, Cambridge University Press, Cambridge, 1998.

[22] Shi-Jun Zhou, Xi Zhu, Analysis of effect of dead loads on natural frequencies of beams using finite-element techniques, Journal of Structural Engineering $112(5)(1996) 512-516$.

[23] Jing Zhang, Wei-Xin Ren, A dynamical stiffness method for determining natural frequencies of beams with allowance for dead load, International Journal of Structural Stability and Dynamics 9 (4) (2009) 777-790.

[24] K.T. Danielson, A.K. Noor, Three-dimensional finite analysis in cylindrical coordinates for nonlinear solid mechanical problems, Finite Elements in Analysis and Design 27 (1997) 225-249.

[25] J. So, Three-dimensional vibration analysis of elastic bodies of revolution, Doctoral dissertation, The Ohio State University (1993) 48. 